\title{
Discovery of Aptamers Targeting Receptor-Binding Domain of the SARS-CoV-2 Spike Glycoprotein
}

\author{
Yanling Song, ${ }^{\text {a }}$ Jia Song, ${ }^{\mathrm{b}}$ Xinyu Wei, ${ }^{\mathrm{a}}$ Mengjiao Huang, ${ }^{\mathrm{a}}$ Miao Sun, ${ }^{\mathrm{a}}$ Lin Zhu, ${ }^{\mathrm{a}}$ Bingqian Lin, ${ }^{\mathrm{a}}$ Haic- \\ ong Shen, ${ }^{a}$ Zhi Zhu, ${ }^{a}$ Chaoyong Yang*a,b \\ a. The MOE Key Laboratory of Spectrochemical Analysis and Instrumentation, State Key Laboratory of Physical Chemistry \\ of Solid Surfaces, Department of Chemical Biology, College of Chemistry and Chemical Engineering, Xiamen University, \\ Xiamen, 361005, China \\ b. Institute of Molecular Medicine, Renji Hospital, School of Medicine, Shanghai Jiao Tong University, Shanghai, 200127, \\ China \\ KEYWORDS: SARS-CoV-2 (2019-nCoV), Aptamer, Receptor-Binding Domain, Spike Glycoprotein
}

\begin{abstract}
The World Health Organization has declared the outbreak of a novel coronavirus (SARS-CoV-2 or 2019-nCoV) as a global pandemic. However, the mechanisms behind the coronavirus infection are not yet fully understood, nor are there any targeted treatments or vaccines. In this study, we identified high-binding-affinity aptamers targeting SARS-CoV-2 RBD, using an ACE2 competition-based aptamer selection strategy and a machine learning screening algorithm. The $\mathrm{K}_{\mathrm{d}}$ values of the optimized CoV2RBD-1C and CoV2-RBD-4C aptamers against RBD were $5.8 \mathrm{nM}$ and $19.9 \mathrm{nM}$, respectively. Simulated interaction modeling, along with competitive with experiments, suggests that two aptamers may have partially identical binding sites at ACE2 on SARS-CoV-2 RBD. These aptamers present an opportunity for generating new probes for recognition of SARS-CoV-2, and could provide assistance in the diagnosis and treatment of SARS-CoV-2 while providing a new tool for in-depth study of the mechanisms behind the coronavirus infection.
\end{abstract}

The novel coronavirus (SARS-CoV-2 or 2019-nCoV) is spreading rapidly around the world and infecting people, causing respiratory illness (named COVID-19), ${ }^{1,2}$ and leading to tens of thousands of deaths. Compared to SARS-CoV, SARSCoV-2 appears to spread more easily via person-to-person contact, which contributed to the WHO's (World Health Organization) declaration of a global pandemic on 11 March 2020. This is the first WHO-declared pandemic caused by a coronavirus.

Several researchers have confirmed that SARS-CoV-2 infects human respiratory epithelial cells using the same receptor as SARS-CoV, angiotensin-converting enzyme II (ACE2). ${ }^{2-4}$ At the outset of the infection, the receptor-binding domain (RBD) of SARS-CoV-2 spike glycoprotein (S) interacts with ACE2 expressed on host cells. Since the function of RBD in $\mathrm{CoV} \mathrm{S}$ protein is indispensable, it responsible for determining the range of the hosts, the specificity of the virus, and the mortality rate. This makes the RBD of SARS-CoV-2 spike glycoprotein a key target for the diagnosis, treatment, and vaccination of SARS-CoV-2.

Although the overall structural homology and the same receptor between SARS-CoV S and SARS-CoV-2 S protein, it has been reported that the three published SARS-CoV RBDdirected monoclonal antibodies do not bind to SARS-CoV-2 RBD. $^{2}$ Thus, there is an urgent need to identify efficient recognition molecules for SARS-CoV-2 RBD. While antibodies have been widely applied in both biology and clinical medicine, the application of aptamers-nucleic acid recognition molecules possess several advantageous traits ${ }^{5-7}$ for the research and treatment of SARS-CoV-2. The smaller size of aptamers (about 2-3 nm in diameter), as compared to antibod- ies (about 12-15 $\mathrm{nm}$ in diameter), subject them to less steric hindrance on the surface of coronavirus (about $100 \mathrm{~nm}$ in diameter). In theory, the smaller size allows for the binding of more recognition molecules on the same surface area of coronavirus. Due to the chemical nature of nucleic acid, aptamers can be chemically synthesized, precisely modified, high thermally stable, and possess little batch-to-batch variation. These traits make for convenient transportation, storage, and standardization. Additionally, aptamers can be combined with other technologies to expand their performance and applications. ${ }^{8-11}$

In this study, we identified aptamers targeting SARS-CoV-2 RBD using an ACE2 competition-based selection strategy and a machine learning screening algorithm. Among the aptamer candidates, two sequences (CoV2-RBD-1 and CoV2-RBD-4) were optimized in length, resulting in hairpin-structured 51base CoV2-RBD-1C and 67-base CoV2-RBD-4C aptamers. The optimized aptamers possess a high binding affinity against SARS-CoV-2 RBD, with respective $\mathrm{K}_{\mathrm{d}}$ values of $5.8 \mathrm{nM}$ and $19.9 \mathrm{nM}$. The results of molecular dynamics simulations (MDS) and experiments on competition suggest that aptamers bind to several amino acid residues of RBD that are key to ACE2 binding. Therefore, aptamers have the potential to inhibit or block the binding of SARS-CoV-2 RBD to ACE2, properties that could be used in the treatment of and prevention against SARS-CoV-2.

\section{EXPERIMENTAL SECTION}

SELEX Procedures. We performed the aptamer selection procedure for SARS-CoV-2 RBD in a manner similar to our previous work. ${ }^{12}$ The initial ssDNA library consisted of a 40-nt 
randomized region and two flanking regions as PCR primer (5'- ATCCAGAGTGACGCAGCA - 40N - TGGACACGGTGGCTTAGT-3'). Initially, 5 nmol of ssDNA library was dissolved in a $500 \mu \mathrm{L}$ binding buffer (PBS with $0.55 \mathrm{mM}$ $\mathrm{MgCl}_{2}$ ) and annealed at $95^{\circ} \mathrm{C}$, after which they were immediately cooled on ice for $5 \mathrm{~min}$ and then at $25^{\circ} \mathrm{C}$ for $10 \mathrm{~min}$. After the RBD-Protein A-beads were incubated with the library at $25^{\circ} \mathrm{C}$ for $30 \mathrm{~min}$, the supernatant was removed and the recovered beads were washed twice in a binding buffer. The revered RBD-Protein A-beads were then added into a PCR mixture (forward primer, biotin-labeled reverse primer, dNTPs, Taq DNA polymerase, and PCR buffer) to amplify the target bound sequences. To obtain a single-stranded library, the PCR product was incubated with streptavidin-coated sepharose beads (GE Healthcare), and denatured by $0.1 \mathrm{M} \mathrm{NaOH}$ for 1 minute. After desalting with $3 \mathrm{~K}$ ultrafiltration tubes (Millipore), the product was used as the library in the next round.

Twelve rounds of selection were performed, and the selection pressure was gradually increased as follows: 1) the amount of library was reduced from 5 nmol to 200 pmol; 2) the incubation time with RBD-Protein A-beads decreased from $30 \mathrm{~min}$ to $8 \mathrm{~min}$; 3) the amount of RBD protein input decreased; 4) from the third round of selection, Protein A-beads and $\mathrm{IgG}$-beads were used as the negative targets in counter selection; 5) after obtaining the DNA-RBD- Protein A-beads from the ninth round of selection, $5 \mu \mathrm{g}$ ACE2 was added and incubated for $30 \mathrm{~min}$, while the supernatant was collected for amplification.

SMART-Aptamer V2.0. SMART-Aptamer V2.0 was developed based on SMART-Aptamer V1.0 $0^{13}$ by redefining the MDA-score in the multidimensional evaluation process. After clustering the aptamer family based on the BLAST all-vs-all and MCL (Markov 227 Cluster) system, we characterized the variation of family size across the sequenced SELEX pools. The predicted change trends (expansion or contraction) between specific SELEX pools were predefined as " $T$ ", according to the experiment design. We used $T_{k}>0$ and $T_{k}<0$ to represent the respective trends of family expansion and contraction. $\mathrm{T}_{\mathrm{k}}$ represents the changing trend between the $\mathrm{k}^{\mathrm{th}}$ and $\mathrm{k}-1^{\text {th }}$ sequenced pools. A " $\mathrm{C}$ " coefficient was defined as follows:

$$
\begin{gathered}
C_{k, i}=\frac{\left(\frac{\text { family_size }_{k, i}}{\text { pool_size }_{k}} \frac{\text { family_size }_{k 1, i}}{\text { pool_size }_{k 1}}\right)}{\frac{\text { family_size }_{k 1, i}}{\text { pool_size }_{k 1}}} \\
\hat{C}_{k, i}=C_{k, i} T_{k}
\end{gathered}
$$

Here, family_size $k, i$ represents the family size of family "i" in the $\mathrm{k}^{\text {th }}$ SELEX pool and pool_size $\mathrm{k}_{\mathrm{k}}$ represents the total sequenced sequences in the $\mathrm{k}^{\text {th }}$ pool, where a clear change in trends has been predefined. Once $\left|\mathrm{C}_{\mathrm{k}, \mathrm{i}}\right|>0.15$ and $\hat{\mathrm{C}}_{\mathrm{k}, \mathrm{i}}<0$, the MDA-Score of the aptamer family "i" was redefined as:

$$
\text { MDA-score }_{i}=\text { MDA-score Pscore }
$$

MDA-score ${ }_{i}$ represents the predicted target-binding probability of aptamer family "i”. The default Pscore was set to 0.8 .

\section{RESULTS AND DISCUSSION}

Enrichment of DNA Library against SARS-CoV-2 RBD

We chose recombinant SARS-CoV-2 RBD expressed with the $\mathrm{Fc}$ region of mouse $\mathrm{IgG}$ at the $\mathrm{C}$-terminus as the aptamer selection target. The SARS-CoV-2 RBD was modified to protein A-beads forming RBD-Protein A-beads via the interaction of IgG-tag epitope and protein A, properly displaying the RBD domain on the micro-spheres, while the protein A-beads and IgG modified protein A-beads (IgG-beads) were used as negative controls from the third round of selection. The first eight rounds of screening followed classic protein SELEX protocols to ensure that the DNA strands with SARS-CoV-2 RBD binding capacity amplify to multiple copies. After 8 rounds of standard selection procedures, we included ACE2 competition in the next 4 rounds of selection (Scheme, Figure 1A). After the DNA library was incubated with RBD-beads, the unbound or weakly bound sequences in the supernatant were removed. ACE2 was then added and incubated with the RBD-beads to collect the sequences that were ACE2-competitive (Scheme). The four subsequent rounds introduced ACE2 and were used to simulate the infection process, favoring the aptamers against RBD with binding sites similar to ACE2. This method can further exclude sequences interacting with the IgG or protein A.

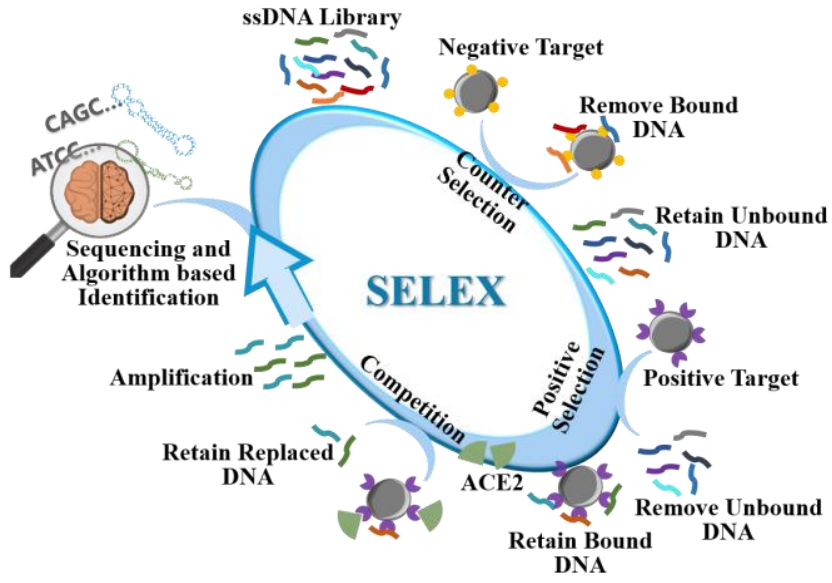

Scheme. Aptamers selection against RBD of SARS-CoV-2 spike glycoprotein.

We selected His-tag RBD-modified Ni-beads (RBD-Nibeads) to monitor the enrichment progress and confirm that the enriched libraries only interact with RBD, and not with Protein A or IgG. Results from flow cytometry revealed, a clear increasing trend of fluorescence in the library binding against RBD-Ni-beads as selection cycles progressed (Figure 1B). Additionally, 12th library also showed stronger fluorescence signal against $\mathrm{F}_{\mathrm{C}}$ tagged $\mathrm{RBD}$ than initial library (SI, Figure S1). However, we observed no increase in fluorescence intensity for Ni-beads (Figure 1C). These results indicated that after 12 rounds of selection, the DNA library was successfully enriched with sequences specifically recognizing SARS-CoV2 RBD.

\section{Aptamer Identification by SMART-Aptamer V2.0}

The development and adoption of high-throughput sequencing (HTS) technology provides billions of candidate sequences, making it increasingly possible to identify high-affinity aptamers. ${ }^{14,15}$ However, there is still a lack of tools that can accurately, effectively, and rapidly identify high-performance aptamers from large sets of sequence data. In order to efficiently discover high-performance aptamers and study the evolutionary lineage using changeable selection pressure, we developed a Sequential Multidimension Analysis algoRiThm V2.0 (SMART-Aptamer V2.0), which is based on our previous work and accounts for ACE2 competitive pressure. 


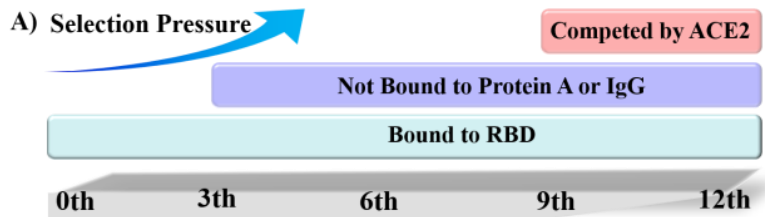

Library Diversity
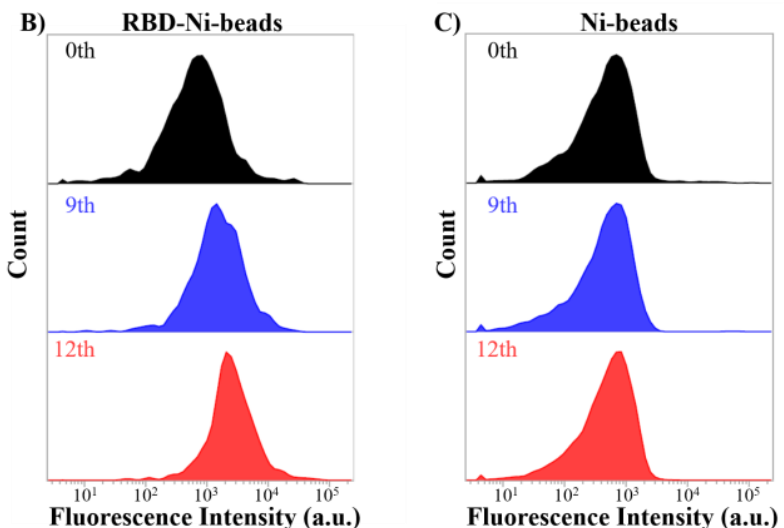

Fig 1. (A) Schematic diagram of the pressure change process with the number of selection rounds. Flow cytometry to monitor the binding increment of enriched pools with (B) RBD-Nibeads (target beads) and (C) Ni-beads (control beads).

The HTS data of the $7^{\text {th }}, 9^{\text {th }}$, and $12^{\text {th }}$ libraries were chosen as inputs. Like our previous SMART-Aptamer V1.0, ${ }^{13}$ three scores were calculated, Kscore, Fscore, and Sscore, to respectively assess the enrichment of motif/substructure, the abundance of aptamer families, and the stability of the overall secondary structure. An MDA-score was then generated by converting these multidimensional metrics into a final metric. We use the SMART-Aptamer integrated an unsupervised machine learning process (Markov Cluster Algorithm, MCL) ${ }^{16}$ to identify the aptamer families and trace the dynamics of the aptamer family size. This assists in the further integration of metrics based on family size. We also added Pscore, a metric based on family size, after considering the new evolutionary pressure of ACE2 competition added in the 9th round. This helped modify the MDA-score to achieve the final MDA-score. Pscore is a penalty score designed to punish aptamer families displaying opposite changes of family size in response to the variation of selection pressure. As far as we know, this is the first in silico tool designed for a non-conventional SELEX process, which we expected extend to aptamer screening for the competition of antibodies, receptors, ligands, and others

SMART-Aptamer V2.0 then calculated the Kscore, Fscore, Sscore, Pscore, and MDA-score of each family. Six representative sequences from six aptamer families were selected from the ten families with the highest MDA-scores, including the family with the highest MDA-score (Figure 2A, Table S2). We synthesized representative sequences (Table S1) from six families with fluorescent labels, and investigated their binding performance. All of the six candidate sequences display stronger binding capabilities toward SARS-CoV-2 RBD compared to the initial library (Figure 2B).

We then chose two representative aptamers with different histories of family expansion and contraction to further characterize and optimize our selection and ensure we had aptamers with varied characteristics. Family 1 displayed an obvious expansion trend from 9-12 rounds (the sequence number increases by $320 \%$, Figure $2 \mathrm{~A} \& \mathrm{C}$ ), while Family 5 remained largely unchanged (the sequence number decreases by about
$14 \%$, Figure 2A\&E). Both sequences have high binding ability against SARS-CoV-2 RBD, and the dissociation constant values $\left(\mathrm{K}_{\mathrm{d}}\right)$ of the CoV2-RBD-1 aptamer (the representative sequence of Family 1) while the CoV2-RBD-4 aptamer (the representative sequence of Family 5) were $3.1 \mathrm{nM}$ and 13.6 $\mathrm{nM}$, respectively (Figure $2 \mathrm{D} \& \mathrm{~F}$ ). These results suggest that SMART-Aptamer V2.0 functions for HTS data during a process with multiple, varied selection pressures.
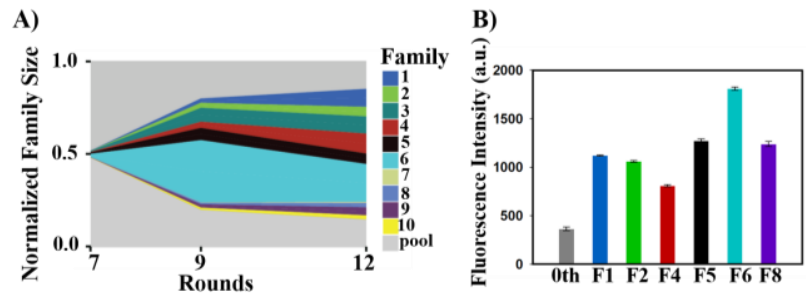

C)

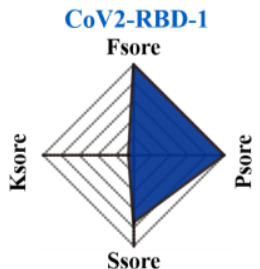

E)

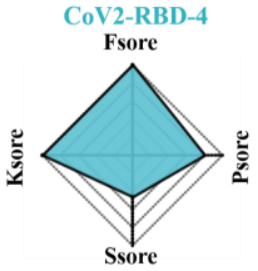

D) $\overparen{\partial}$

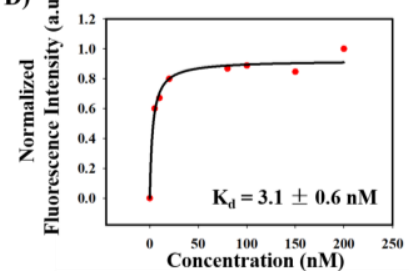

F)

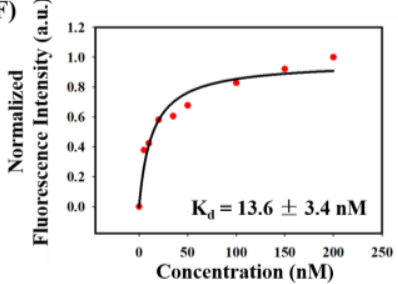

Fig 2. (A) Muller diagram shows the dynamic of the sizes of 10 verified target-binding aptamer families across three rounds $\left(7^{\text {th }}, 9^{\text {th }}\right.$, and $\left.12^{\text {th }}\right)$ of SELEX pools. The total number of sequenced aptamers of each round was normalized to 1. (B) Flow cytometry to investigate the binding performance of representative sequences from six families randomly selected from the ten families with the highest MDA-scores against RBD. (C) \& (E) Radar map of scores of CoV2-RBD-1 aptamer (C) and CoV2-RBD-4 aptamer (E). The binding affinity of CoV2-RBD-1 aptamer (D) and CoV2-RBD-4 aptamer (F) against RBD-Ni-beads.

\section{Optimization of the Selected Aptamer}

Typically, not all nucleotides are essential for target binding: a shorter sequence reduces interference from the steric effect and the cost of synthesis. We retained the whole structure of the CoV2-RBD-1 aptamer by removing the bases of both terminals that were not involved in forming the stem-loop structure, yielding a 51-nt 3-hairpin-structured CoV2-RBD-1C aptamer (Fig. 3A, 5' CAGCACCGACCTTGTGCTTTGGGAGTGCTGGTCCAAG GGCGTTAATGGACA-3'). We then evaluated the binding capability and specificity of the truncated aptamer CoV2RBD-1C against SARS-CoV-2 RBD. For RBD-his-beads, the fluorescence intensities of the CoV2-RBD-1C aptamer were significantly stronger than in the initial library, while for Nibeads the aptamer intensities were similar to that of the initial library (Figure 3B). We determined that the $\mathrm{K}_{\mathrm{d}}$ value of the CoV2-RBD-1C aptamer against RBD was $5.8 \pm 0.8 \mathrm{nM}$ (Figure 3C), similar to that of the full-length CoV2-RBD-1 aptamer. We evaluated the binding performance of the selected aptamer against SARS-CoV-2 RBD in $80 \%$ human plasma, to explore the practical application value of the selected aptamer. 
No obvious decrease in the amount of aptamer binding to the target RBD in $80 \%$ plasma condition was observed, compared to the buffer (Figure 3D). Similarly, the CoV2-RBD-4 sequence was optimized, yielding a 67-nt hairpin sequence CoV2-RBD-4C (Figure 3E, 5'ATCCAGAGTGACGCAGCATTTCATCGGGTCCAAAAGG GGCTGCTCGGGATTGCGGATATGGACACGT-3'). Further analysis of binding affinity revealed a $\mathrm{K}_{\mathrm{d}}$ of $19.9 \pm 2.6 \mathrm{nM}$ for CoV2-RBD-4C (Figure 3G). Under 80\% plasma incubation

A)

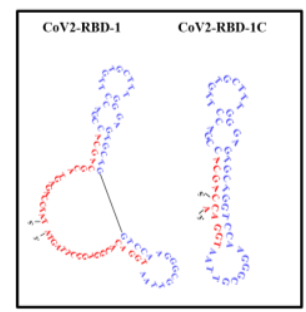

E)

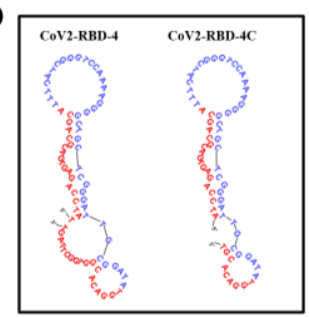

B)
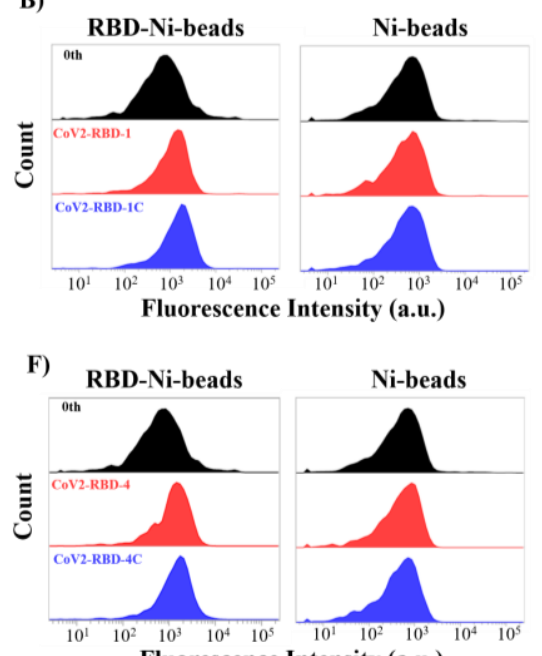

condition, approximately $75 \%$ of binding aptamers were maintained compared to the buffer (Figure 3H). These results demonstrated that the two aptamers not only bind to SARSCoV-2 RBD with high affinity in a buffer solution, but could also recognize $\mathrm{RBD}$ in complex environments, making them promising for practical applications. In addition, the two aptamers can also recognize the spike glycoprotein of SARSCoV-2 (Figure S4), suggesting that they may be used for $\mathrm{S}$ protein recognition expressed on SARS-CoV-2.
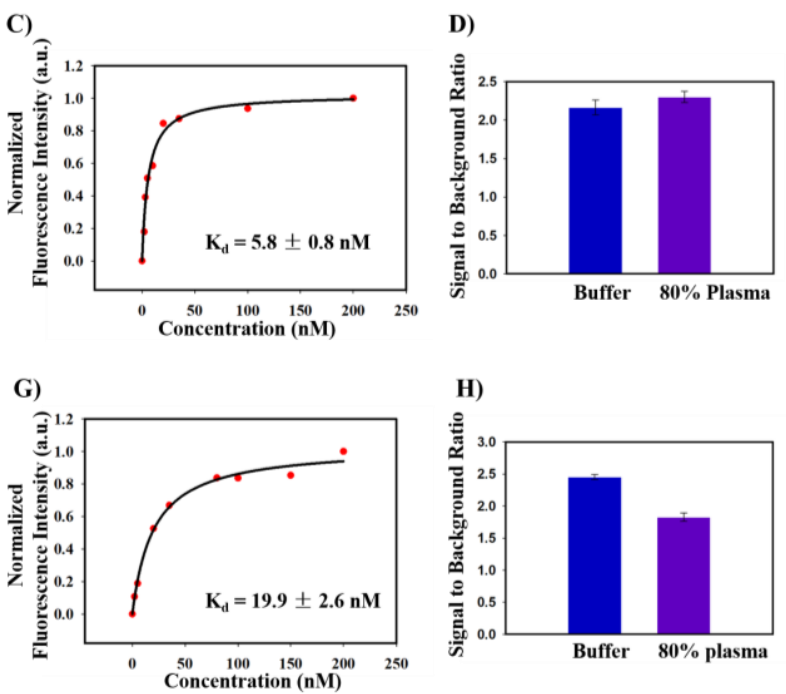

Figure 3. The secondary structures of CoV2-RBD-1 and CoV2-RBD-1C aptamers (A), and CoV2-RBD-4 and CoV2-RBD-4C aptamers (E), which were predicted using Mfold. Flow cytometric analysis of CoV2-RBD-1C (B) and CoV2-RBD-1C (F) binding to target beads (RBD-Ni-beads) and control beads (RBD-Ni-beads). Binding curves of CoV2-RBD-1C aptamer (C) and CoV2-RBD4C (G) against RBD. (D) The signal/background ratios of CoV2-RBD-1C aptamer (D) and CoV2-RBD-1C aptamer (H) against RBD in buffer and $80 \%$ plasma.

Molecular Docking and Molecular Dynamic Simulations

To better understand the interaction patterns between SARS-CoV-2 RBD and the two aptamers, we performed molecular docking and molecular dynamics simulations (MDS). We first transformed the three-dimensional structure of equivalent ssRNA of the aptamer from the secondary structure generated by mfold software using RNAcomposer. After replacing $\mathrm{U}$ with $\mathrm{T}$ base in the RNA structure, MDS were performed to refine the three-dimensional structures of the two aptamers. Meanwhile, we obtained the three-dimensional structure of the SARS-CoV-2 S protein with a single RBD from the RCSB PDB data bank (ID: 6VSB). ${ }^{2}$ Molecular dockings were then performed to model the structures of SARSCoV-2 S protein-aptamer complexes, which were used as starting points for MDS. We obtained final average structures of the interaction complexes based on 5000 snapshots, which were extracted from the last $10 \mathrm{~ns}$ trajectory of MDS.

The resulting SARS-CoV-2 S protein and CoV2-RBD-1C contain two consecutive binding interfaces (Figure 4A). The binding interface near the 3' terminal of aptamer, T42, T43, and T43 of the CoV2-RBD-1C form a network of hydrogen bonds with Thr500, Gln506, and Asn437 from RBD (Figure $4 \mathrm{~B})$. This contact cluster between the aptamer and RBD is very close to that of one end of the "bridge" interaction between ACE2 and RBD (Gln498, Thr500, and Asn501) ${ }^{4}$; they also have the same binding amino acid of RBD (Thr500). The contact model of CoV2-RBD-4C aptamer and RBD has a binding interface (Figure 4D) and C53, G54, and A56 of the CoV2-RBD-4C form a network of hydrogen bonds with Gln409, Lys417, and Try421 from RBD (Figure 4F). This binding interface shares the same binding amino acid of RBD
(Lys417) as the ACE2-RBD binding complex, which is in the middle segment of the ACE2-RBD "bridge" interaction".

To validate the target-binding sites suggested by MDS, we proposed 5 mutants of CoV2-RBD-1C (Figure 4C) and $3 \mathrm{mu}-$ tants of CoV2-RBD-4C (Figure 4G) by deleting some suggested binding sites. Flow cytometry results indicated that all the mutants showed weaker binding performance against RBD-Ni-beads than original aptamers (Figure 4C, G). This result indicated that, MDS helps us to understand binding patterns at the molecular level of the two aptamers targeting RBD, and suggest two aptamers may have partially identical binding sites, like ACE2 on SARS-CoV-2 RBD. Further study of the binding surface/sites between RBD and aptamers can help improve the relationship and enhance competitiveness with ACE2.

To further study the competition between aptamers and ACE2, ACE2 was added into the SARS-CoV-2 RBD-aptamer complexes. Even when the amount of ACE2 was five times that of the aptamer, CoV2-RBD-1C aptamer was replaced by $44 \%$ (Figure 4D) of aptamers binding on RBD, while CoV2RBD-4C was replaced by $56 \%$ (Figure $4 \mathrm{H}$ ). The moderate competitive ability of ACE2 to RBD-binding-aptamers suggests that ACE2 and related aptamers could partially share the same binding sites, which is consistent with the results of our simulated interaction models. In addition, since the $\mathrm{K}_{\mathrm{d}}$ value of CoV2-RBD-1C against SARS-CoV-2-RBD is $5.5 \mathrm{nM}$ and that of CoV2-RBD-4C is $19.9 \mathrm{nM}$, which is less than the reported $\mathrm{K}_{\mathrm{d}}$ of ACE2 against SARS-CoV-2 RBD (34.6 nM), ${ }^{2}$ incomplete competition is reasonable. Both the simulation and experimental results indicate that the two aptamers and ACE2 may have a competitive relationship, meaning the two ap- 
tamers could be applied to the diagnosis, prevention, and
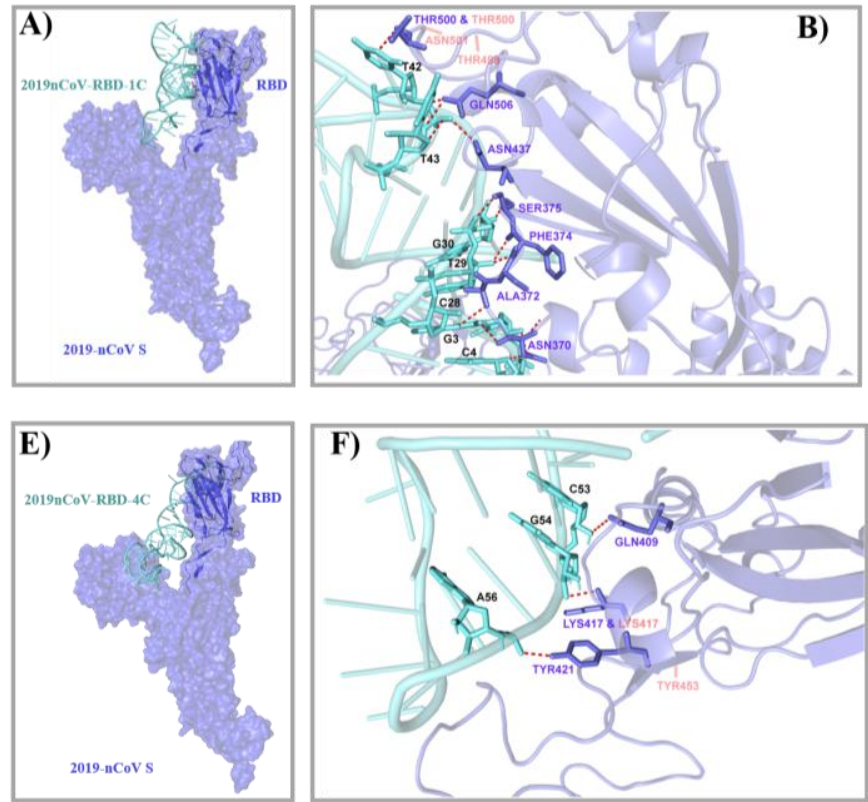

treatment against SARS-CoV-2.
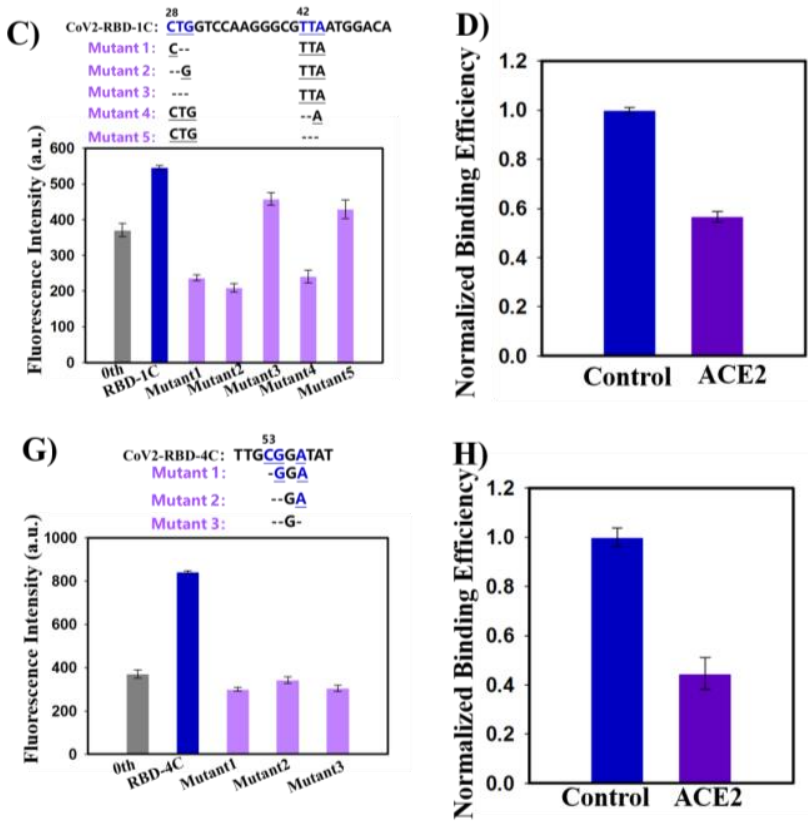

Fig 4. (A) The overall structures of the CoV2-RBD-1C aptamer (cyan) and the SARS-CoV-2 S protein complex (blue), (E) and the CoV2-RBD-4C aptamer (cyan) and the SARS-CoV-2 S protein complex (blue). (B) Detailed analysis of the interface between CoV2-RBD-1C and RBD, (F) and the interface between CoV2-RBD-4C and RBD. Hydrogen bonds are shown by red, dashed lines. The amino acids of SARS-CoV-2-RBD targeted by aptamers are shown in blue; and the amino acids of SARS-CoV-2-RBD targeted by ACE2 are shown in red. (C) \&(G) Flow cytometry results show that mutants with binding sites deleted exhibited significantly lower binding performance against RBD-Ni-beads compared to (C) CoV2-RBD-1C or (G) CoV2-RBD-4C aptamer. The lines represent the bases that were deleted. (D) $\&(\mathrm{H})$ The normalized binding efficiency of aptamers against RBD, under control or competition by ACE2. (D) for CoV2-RBD-1C aptamer and (H) for CoV2-RBD-4C.

\section{CONCLUSIONS}

We used the SARS-CoV-2 RBD as a target for the development of serial DNA aptamers using ACE2 competition and a machine learning screening algorithm. By retaining the main motif of the full-length aptamers, we optimized two aptamers (with $\mathrm{K}_{\mathrm{d}}$ values of $5.8 \mathrm{nM}$ and $19.9 \mathrm{nM}$ ) against SARS-CoV-2 RBD. The selected aptamers are ideal recognition probes for SARS-CoV-2 RBD due to their high affinity, their small size, and their ease of modification and use. These properties make them ideal for performing diagnostic and basic research on the properties of SARS-CoV-2. Our simulation and experimental results both suggest that the aptamers and ACE2 could have a competitive relationship. Therefore, aptamers are candidates for preventing the SARSCoV-2 from infecting human cells and will be increasingly applied to resist viral infections and antiviral therapeutics.

\section{ASSOCIATED CONTENT}

\section{Supporting Information}

Supporting figures, tables, flow cytometry assay, molecular docking and dynamic simulations.

\section{AUTHOR INFORMATION}

\section{Corresponding Author}

\footnotetext{
*ylsong@xmu.edu.cn

* cyyang@xmu.edu.cn
}

\section{Notes}

The authors declare no competing financial interests.

\section{ACKNOWLEDGMENT}

We are grateful for the Fundamental Research Funds for the Central Universities (20720200041) for their financial support.

\section{Author Contributions}

The manuscript was written through contributions of all authors. C.Y.Y. and Y.L.S. conceived of this project and improved the manuscript. Y.L.S. designed the experiment, performed the analysis and wrote the manuscript. J.S. and X.Y.W. performed the part of experiments. All authors read and approved the final manuscript.

\section{Notes}

The authors declare no competing financial interest

\section{REFERENCES}

(1) Gorbalenya, A.E., Baker, S.C., Baric, R.S. et al. Nat. Microbiol. 2020, doi: $10.1038 / \mathrm{s} 41564-020-0695-\mathrm{z}$

(2) Wrapp D., ang N., Corbett K. S, Goldsmith J. A., Hsieh C. L., Abiona O., Graham B. S., McLellan J. S, Science 2020, 6483, 1260-1263

(3) Walls A. C., Park Y.-J., Tortorici M. A., Wall A., McGuire A. T., Veesler D., Cell, 2020, doi:10.1016/j.cell.2020.02.058

(4) Yan R. H., Zhang Y. Y., Y Li., Xia L., Guo Y.Y., Zhou Q., Science 2020, doi: 10.1126/science.abb2762 
(5) Teng, I.; Li, X.; Yadikar, H.; Yang, Z.; Li, L.; Lyu, Y.; Pan, X.; Wang, K. K. W.; Tan, W. J. Am. Chem. Soc. 2018, 140, 14314-14323.

(6) Wang, L.; Bing, T.; Liu, Y.; Zhang, N.; Shen, L.; Liu, X.; Wang, J.; Shangguan, D. J. Am. Chem. Soc. 2018, 140, 18066-18073.

(7) Huang M. J.; Yang J.J.; Wang T.; Song J.; Xia J. L.; Wu L. L.; Wang W.; Wu Q. Y.; Zhu Z.; Song. Y. L.; Yang C. Y.; Angew 2020, $59,4800-4805$.

(8) Shen, Q.; Xu, L.; Zhao, L.; Wu, D.; Fan, Y.; Zhou, Y.; OuYang, W.-H.; Xu, X.; Zhang, Z.; Song, M.; Lee, T.; Garcia, M. A.; Xiong, B.; Hou, S.; Tseng, H.-R.; Fang, X. Adv. Mater. 2013, 25, 2368-2373.

(9) Liu X. G.; Zhang F.; Jing X. X.; Pan M. C.; Liu, P.; Li, W.W.; Zhu, B. W.; Li, J.; Chen, H.; Wang, L. H.; Lin, J. P.; Liu, Y.; Zhao, D. Y.; Yan, H.; Fan, F. H. Nature. 2018, 559, 593-598.

(10) Liu, M.; Chang, D.; Li, Y. F. Acc. Chem. Res. 2017, 50, 2273-2283.
(11) Li, F.; Lu, J.; Liu, J.; Liang, C.; Wang, M.; Wang, L.; Li, D.; Yao, H.; Zhang, Q.; Wen, J.; Zhang, Z.-K.; Li, J.; Lv, Q.; He, X.; Guo, B.; Guan, D.; Yu, Y.; Dang, L.; Wu, X.; Li, Y.; Chen, G.; Jiang, F.; Sun, S.; Zhang, B.-T.; Lu, A.; Zhang, G. Nat. Commun. 2017, 8, 1390.

(12) Song, Y.; Zhu, Z.; An, Y.; Zhang, W.; Zhang, H.; Liu, D.; Yu, C.; Duan, W.; Yang, C. J. Anal. Chem. 2013, 85, 4141-4149.

(13) Song, J.; Zheng, Y.; Huang, M. J.; Wu, L. L.; Wang, W.; Zhu, Z.; Song, Y. L.; Yang, C. Y. Anal. Chem. 2020, 92, 3307-3314.

(14) Phuong, D.; Hoinka, J.; Takahashi, M.; Zhou, J.; Ho, M.; Wang, Y.; Costa, F.; Rossi, J. J.; Backofen, R.; Burnett, J.; Przytycka, T. M., Cell Syst. 2016, 3, 62-70.

(15) Kinghorn A. B.; Dirkzwager R. M.; Liang S. L.; Cheung Y.-W.; Fraser L. A.; Shiu S. C.; Tang M.S. L.; Tanne J. A. Anal. Chem. 2016, 88, 6981-6985.

(16) Enright, A. J.; Van Dongen, S.; Ouzounis, C. A. Nu cleic Acids Res. 2002, 30, 1575-1584 
Table of Contents artwork

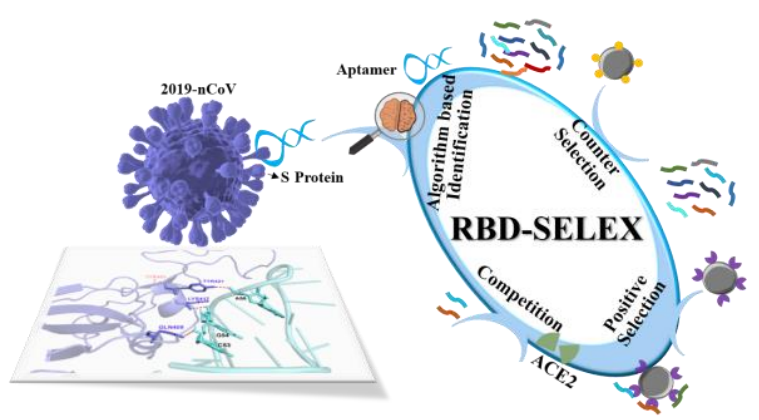

